\title{
Far-ultraviolet Cooling Features of the Antlia Supernova Remnant
}

\author{
Jong-Ho Shinn ${ }^{1 \dagger}$, Kyoung Wook Min ${ }^{1}$, Ravi Sankrit ${ }^{3}$, Kwang-Sun Ryu ${ }^{1}$, Il-Joong Kim ${ }^{1}$, \\ Wonyong $\mathrm{Han}^{2}$, Uk-Won Nam², Jang-Hyun Park ${ }^{2}$, Jerry Edelstein ${ }^{3}$, and Eric J. Korpela ${ }^{3}$ \\ jhshinn@kaist.edu
}

\begin{abstract}
We present far-ultraviolet observations of the Antlia supernova remnant obtained with Far-ultraviolet IMaging Spectrograph (FIMS, also called SPEAR). The strongest lines observed are C IV $\lambda \lambda 1548,1551$ and C III $\lambda 977$. The C IV emission of this mixed-morphology supernova remnant shows a clumpy distribution, and the line intensity is nearly constant with radius. The C III $\lambda 977$ line, though too weak to be mapped over the whole remnant, is shown to vary radially. The line intensity peaks at about half the radius, and drops at the edge of the remnant. Both the clumpy distribution of $\mathrm{C} I V$ and the rise in the $\mathrm{C} \mathrm{IV}$ to $\mathrm{C}$ III ratio towards the edge suggest that central emission is from evaporating cloudlets rather than thermal conduction in a more uniform, dense medium.
\end{abstract}

Subject headings: ISM: individual (Antlia SNR) — ISM: individual (Gum Nebula) - ISM: individual (Vela SNR) - (ISM:) supernova remnants - ultraviolet: ISM

\section{Introduction}

The Antlia supernova remnant $(\mathrm{SNR})$ is a nearby $\left(d_{A} \simeq 60-340 \mathrm{pc}\right)$, large $\left(\sim 24^{\circ}\right.$ in diameter), old $\left(t_{A} \sim 1 \mathrm{Myr}\right)$ SNR recently discovered by McCullough et al. (2002), centered

\footnotetext{
${ }^{1}$ Korea Advanced Institute of Science and Technology (KAIST), 373-1 Guseong-dong, Yuseong-gu, Daejeon, 305-701, South Korea

${ }^{2}$ Korea Astronomy and Space Science Institute (KASI), 61-1 Hwaam-dong Yuseong-gu Daejeon, 305-348, South Korea

${ }^{3}$ Space Sciences Laboratory, University of California, Berkeley, CA 94720

${ }^{\dagger}$ Dept. of Physics and Astronomy, Seoul National University, San 56-1 Shillim9-dong, Gwanak-gu, Seoul, 151-747, South Korea
} 
on $(l=276.52, b=+19.05)$. It shows a center-filled X-ray morphology, which is partially enclosed by prominent $\mathrm{H} \alpha$ and weak radio features (cf. Fig. 1). The remnant abuts the Gum Nebula to its southwest, and the boundary lies along the radio ridge seen in Fig. 1. Based on the X-ray and radio morphologies, we classify the Antlia SNR as a mixed-morphology (MM) SNR (Rho \& Petre 1998). The X-ray is clearly center-filled, and though the radio shell is weak, there is no central radio emission. Furthermore there is no known pulsar near the center of the Antlia SNR so it is unlikely to be pulsar-driven nebula.

To explain the physical characteristics of MM SNRs, in particular their center-filled Xray morphologies, two models have been invoked. Both focus on how the interior density, and thereby the emissivity may be increased. In the first model (White \& Long 1991, hereafter "cloudlet" model), thermal evaporation of small clouds overrun by the supernova shock and engulfed by the hot gas bubble increases the density of the interior gas. In the second model (hereafter "dense medium" model), applied to the SNR W44 by Cox et al. (1999), thermal conduction in a dense medium alone increases the interior density of the remnant as heat is transported outward from the center. These two models assume different distributions of the denser component that would show radiative-cooling features as the remnant evolves.

Here, we report the detection of such cooling features in the far-ultraviolet (FUV) wavelengths from the Antlia SNR. We observed the Antlia SNR with the Far-ultraviolet IMaging Spectrograph (FIMS, also called SPEAR), and detected several emission lines of which C IV $\lambda \lambda 1548,1551$ and C III $\lambda 977$ are the strongest. In the following sections we describe the observations, present the analysis and results, and discuss what we have learnt about MM SNRs.

\section{Observation and Data Reduction}

FIMS/SPEAR is a dual imaging spectrograph aboard the first Korean scientific satellite, STSAT-1, and is uniquely suited to observe the diffuse radiation in the FUV domain (Edelstein et al. 2006b). It has two spectral channels (S channel for 900-1150 A and L channel for $1350-1750 \AA$ ), and their field-of-views are $4.0 \times 4^{\prime} .6$ and $7^{\circ} .4 \times 4^{\prime} .3$, respectively. The spectral and imaging resolutions are, respectively, $\lambda / \Delta \lambda \sim 550$ and $\sim 5^{\prime}$ on both channels. The instrument, its on-orbit performance, and the data processing procedures are described in Edelstein et al. (2006a).

The Antlia SNR was observed during sky surveys between 2004 February 29 and April 19. Some of the observations were performed with the shutter reduced to $10 \%$ of the full slit length, and we also included them in the analysis. Overall, the exposure time varies 
from $\sim 1$ to $\sim 30$ s. We followed the general data processing procedures described in Edelstein et al. (2006a) and then made a two-dimensional array for flexibility in handling the spectral and spatial information. The spectral bin sizes were $0.5 \AA$ and $1.0 \AA$ for the $\mathrm{S}$ and L channels, respectively. The spatial information was encoded by HEALPix pixelization (Górski et al. 2005), and the pixel size was $\sim 27$. $5 \times 27$.5. A larger pixel size $\left(\sim 55^{\prime} .0 \times 55^{\prime} .0\right)$ was used in generating the emission-line map to enhance the signal intensity per pixel (cf. next paragraph). We adopted the effective area estimated in 2004 April for the $\mathrm{S}$ channel data since it is closer to the observation period than the 2003-November one (cf. Edelstein et al. 2006a).

The emission-line map was produced through the following two steps: first, the spectrum of an individual pixel was fitted by a constant continuum plus a center-fixed Gaussian profile, with its width corresponding to the spectral resolution at the line center. The fitting was carried out using a Bayesian method to estimate the signal amplitude in the presence of background assuming the Poisson statistics, as in Seon et al. (2006). This method avoids the over-subtraction, which often occurs when the continuum adjacent to the emission line is used as a model continuum. Then, the emission-line map was smoothed with a Gaussian kernel, whose full-width-half-maximum is 2.5 , to enhance the features of any significance.

\section{Analysis and Results}

Following McCullough et al. (2002), we assumed the boundary of the Antlia SNR to lie roughly along a circle of $24^{\circ}$ in diameter (cf. Fig. 1). This boundary is defined mainly by the radio shell. To the south, the $\mathrm{H} \alpha$ emission also coincides with this shell. There are $\mathrm{X}$-ray emission features that lie outside this nominal boundary of the remnant. This is not surprising since the direction towards the Antlia SNR is close to the Galactic plane and contains several active emission regions (including the Vela SNR). In this analysis we do not analyze these regions, nor do we explore the possibility that they may be associated with the Antlia SNR.

Fig. 2 shows the total spectrum of the remnant extracted within the circle on Fig. 1, in $\mathrm{CU}\left(\equiv \mathrm{ph} \mathrm{s}^{-1} \mathrm{~cm}^{-2} \mathrm{sr}^{-1} \AA^{-1}\right.$; continuum unit). In order to enhance the diffuse radiation relative to the radiation from point sources, we excluded the pixels of which wavelength-averaged intensity is greater than $10^{5} \mathrm{CU}$ and $10^{4} \mathrm{CU}$ for the $\mathrm{S}$ and L channels, respectively. Several resonant emission lines are evident: C III $\lambda 977$, N I $\lambda 1135$, Si II* $\lambda 1533$, C IV $\lambda \lambda 1548,1551$, and Al II $\lambda 1671$. Si II $\lambda 1527$, a doublet companion of Si II* $\lambda 1533$ and a transition to the true ground state, was not seen in the spectrum, probably due to the resonant scattering by the interstellar warm ionized medium as in previous studies (Shinn et al. 2006; Korpela et al. 


\section{6; Kregenow et al. 2006).}

Table 1 lists the intensities of each identified emission line from the total spectrum in $\mathrm{LU}\left(\equiv \mathrm{ph} \mathrm{s}^{-1} \mathrm{~cm}^{-2} \mathrm{sr}^{-1}\right.$; line unit). The observed intensities were extinction-corrected with $R_{V}=3.1$ Milky Way dust model (Weingartner \& Draine 2001; Draine 2003), adopting the hydrogen column density $N(\mathrm{H} \mathrm{I})=3.0 \times 10^{20} \mathrm{~cm}^{-2}$ McCullough et al. (2002) derived. C IV and C III are the strongest lines and the ones that are associated with the gas in the SNR. The other lines are typically too weak to be produced in shocks or thermal interfaces (e.g. Borkowski et al. 1990) and probably arise in the nearby warm ionized medium due to photoionization. Therefore we focus on these lines from ionized carbon, both of which are strong coolants of gas at around $10^{5} \mathrm{~K}$ (e.g. Cox \& Daltabuit 1971). O VI was not detected in our spectrum, so we present the $90 \%$ upper limit. We note that the confidence interval of $\mathrm{I}(\mathrm{O}$ VI)/I(C III) from our data is consistent with the value found by Danforth et al. (2003) for SNR 0057-7226, an MM SNR in the Small Magellanic Cloud, which they observed with the Far Ultraviolet Spectroscopic Explorer.

Fig. 3 displays the emission-line map of C IV $\lambda \lambda 1548,1551$. The C IV intensity is about 10000 - 20000 LU over the remnant, and its diffuse background, measured over the "BG" box in Fig. 3, is $3620 \pm 724 \mathrm{LU}$. It shows about $3^{\circ}-7^{\circ}$ scale features, which corresponds to $3-40 \mathrm{pc}$ adopting the inferred distance $d_{A}$; however, the minimum scale can be smaller since the resolution of the map is only about $2^{\circ} .5$. It generally shows a clumpy distribution all over the circle, and two intense-peak regions exist at both sides of the elongated hot gas seen in $3 / 4 \mathrm{keV}$ map (see Fig. 1). We could generate the emission-line map only for C IV; the signal strength of C III was not sufficient to generate a map.

Instead, we analyzed the radial variation of $\mathrm{C}$ III intensity along with $\mathrm{C}$ IV intensity. Since both $\mathrm{C}^{2+}$ and $\mathrm{C}^{3+}$ originate from the same atom, but with different ionization energy $\left(E_{\text {ion }}=24 \mathrm{eV}\right.$ for $\mathrm{C}^{2+}$ and $E_{i o n}=48 \mathrm{eV}$ for $\left.\mathrm{C}^{3+}\right)$, their line ratio is a useful diagnostic for the ionization state. We chose subregions as in Fig. 3, and estimated the intensity of C III and C IV. The subregional spectra were extracted from two adjacent subregions to get strong enough signals and simultaneously to see the average variation of the intensity profile. This method is basically similar with the box-car smoothing of the "true" intensity profile. The line intensities were estimated from each spectrum employing a $\chi^{2}$ minimization method (Kriss 1994). The two upper panels of Fig. 4 show the subregional spectra around C III and C IV, respectively. The two lower panels of Fig. 4 show the radial variations of their intensity and relative ratio, I(C IV)/I(C III), respectively. C IV intensity is nearly constant with radius while $\mathrm{C}$ III intensity peaks at about half the radius, making their line ratio minimum near the C III peak. 


\section{Discussion}

The two models for MM SNRs, discussed in $\S 1$, use different distributions of the coldand-dense gas component and therefore differ in their predictions of how the regions of radiative cooling are distributed. The $\mathrm{C}$ IV and $\mathrm{C}$ III emission observed trace this cooling gas, and we now compare our observations to the model predictions.

Shelton et al. (1999) performed a 1-D numerical simulation on a SNR evolving in a dense ambient medium with a density gradient and thermal conduction. Regardless of the thermal conduction mechanism, the $T=10^{5} \mathrm{~K}$ region, where $\mathrm{C}$ IV emission is likely originate, is located almost at the same position - near the edge of the remnant. Such an edge-concentration of C IV was also predicted in the 1-D old-SNR model of Slavin \& Cox (1992), which includes a similar thermal-conduction treatment. Recently, Tilley et al. (2006) modeled the intensity of O VI $\lambda \lambda 1032,1038$, another FUV cooling line, for an MM SNR in 2-D under an anisotropic thermal conduction caused by magnetic fields. Their result also shows an edge-concentration. On the contrary, our C IV map (Fig. 3) shows a clumpy distribution over the remnant and C IV peaks are seen at the central region. This suggests that the "dense medium" model may not be suitable to explain the formation of the Antlia SNR. The C IV detection in the middle of the hot gas (region 3) in another MM SNR, the Lupus Loop, also supports this argument (Shinn et al. 2006; Shinn 2007).

There are no published predictions available for the C IV emission from evaporating cloudlets. However, we can qualitatively compare the expected distribution of C IV emission from such a model with our observations. The clumpy distribution of C IV emission can be explained, if small clouds were inhomogeneously scattered and engulfed by the hot bubble as in the "cloudlet" model. The fact that there are numerous B stars in the vicinity of the Antlia SNR but no known O stars also supports the "cloudlet" model. O-type stars may have sufficient photoionizing radiation and strong stellar wind to clear the molecular material in a region of $\sim 15 \mathrm{pc}$ around them, while early B-type stars are not capable of this and may directly interact with molecular gas when they explode (Chevalier 1999). Thus, if the progenitor of the Antlia SNR were a B-type star, the remnant is likely to include interactions with the surrounding clouds. In addition, the size of C IV features is consistent with the "cloudlet" model. Borkowski et al. (1990) computed the critical cloud radius of $\sim 15 \mathrm{pc}$, below which clouds are evaporating. This scale is in agreement with the one seen in our $\mathrm{C}$ IV map, $3-40 \mathrm{pc}$, given the instrumental imaging resolution.

The $\mathrm{C}$ IV intensity is nearly constant with radius along the direction measured, while the C III intensity drops sharply at the edge (Fig. 4). This suggests that the temperature is probably not decreasing near the edge as expected for the "dense medium" model (Shelton et al. 1999). We note that the gas is probably not in ionization equilibrium, and 
that projection effects could influence the C III to C IV ratio. Thus, the observed trend weakly supports the "cloudlet" model over the "dense medium" model.

In both "cloudlet" and "dense medium" models, the basic physical mechanism producing the FUV emitting gas is thermal conduction. The line intensities expected from such a conduction front have been calculated by Slavin (1989) and Borkowski et al. (1990). The former assumed a stationary interface and an oblique magnetic field including the saturated thermal conduction, while the latter assumed an evolving interface and a perpendicular magnetic field neglecting the saturated thermal conduction. Our observed line ratios between C III and C IV, and O VI and C IV (Table 1) are consistent with the evaporation phase of the Borkowski et al. (1990) models but not with the Slavin (1989) models.

For a single evaporative interface at a pressure, $P / k_{B}=3750 \mathrm{~cm}^{-3} \mathrm{~K}$, the Borkowski et al. (1990) models predict that the C IV doublet intensity will be about $100 \mathrm{LU}$ (see their Table 1). The total intensity scales linearly with the pressure. The interior pressure in a 1 Myr old remnant is about $10000 \mathrm{~cm}^{-3} \mathrm{~K}$ (Slavin \& Cox 1992), and so the expected C IV intensity is about $300 \mathrm{LU}$ per interface. We note that this is the intensity for an interface viewed face-on and that the observed brightness will be higher when viewed closer to edge-on, depending on the width of the emitting region (K. Borkowski, private communication). The detected $\mathrm{C}$ IV intensity (without the contribution of the background C IV) is about 10,000 LU (Table 1, and $\$ 3$ above). This is a factor about 30 higher than produced by a single interface viewed face-on. Geometric enhancement of the emission due to the presence of many interfaces along the line of sight and due to viewing angles is necessary to account for the observed C IV emission. Each cloud contributes two interfaces if viewed close to face-on (one from the front and the other from the back) or a single interface with some amount of "limb-brightening" if viewed close to edge on. Thus, 15 clouds could reproduce the observed C IV emission, but the number could be much lower if the viewing angle is close to edge-on for one or more interfaces. Clearly, if the pressure in the SNR were in fact higher than assumed for this calculation the geometric enhancement factor required would be correspondingly lower.

In astrophysical systems, FUV line emission can be produced in gas cooling from X-ray emitting temperatures and in shocked gas. The first of these mechanisms is not likely for the emission observed from the Antlia SNR. Cooling gas produces strong O VI emission, in general much stronger than C IV (Edgar \& Chevalier 1986), but which is not observed. Shocks with velocities of about $110-130 \mathrm{~km} \mathrm{~s}^{-1}$ would produce line ratios similar to what we observed (Hartigan et al. 1987). The shock model E110 of Hartigan et al. (1987) predicts that the C IV intensity is $36000 \mathrm{LU}$ when the pre-shock density $\left(N_{0}\right)$ is $0.1 \mathrm{~cm}^{-3}$. The line intensity is proportional to pre-shock density, and so the observed $\mathrm{C}$ IV intensity will be produced by a $110 \mathrm{~km} \mathrm{~s}^{-1}$ shock running into gas with $N_{0} \sim 0.03 \mathrm{~cm}^{-3}$. The driving pressure 
for such a shock, $P / k_{B} \sim 36000 \mathrm{~cm}^{-3} \mathrm{~K}$. This is not far above the nominal pressure in a 1 Myr old remnant quoted above. Alternatively, the pre-shock density could be as low as 0.01 $\mathrm{cm}^{-3}$ and a modest geometric enhancement would be sufficient to produce the observed $\mathrm{C}$ IV emission. However, shock-cloud interaction regions exist at the boundary of the remnant. If the C IV emission detected is from the interior, then it is hard to invoke shocks as the exciting mechanism since the shock crossing times are much shorter (e.g. Cowie et al. 1981). If the the emitting regions are close to the boundary and their location near the middle of the remnant (Fig. 3) is a projection effect, then shocks cannot be ruled out as the mechanism for producing the FUV emission. However, as we have shown above, thermal conduction can produce the observed C IV emission although some geometric enhancement is needed given the expected interior pressure in an old remnant such as the Antlia SNR.

\section{Conclusion}

We have presented and analyzed FUV observations of the Antlia SNR obtained with FIMS/SPEAR. The Antlia SNR was identified as an MM SNR based on its X-ray and radio morphology, and its boundary was assumed as a $24^{\circ}$-diameter circle at the center. The clumpy C IV map without an edge-concentration and the radial variation of $\mathrm{I}(\mathrm{C}$ IV $) / \mathrm{I}(\mathrm{C}$ III) within the remnant suggest that the "cloudlet" model, rather than the "dense medium" model, is plausible for the formation of the Antlia SNR's center-filled X-ray morphology.

The Antlia SNR is one of about 25 MM SNRs in the Galaxy, and perhaps the only one that can be studied in ultraviolet - all the others are suffering high extinction. Hence, the results presented here are unique, showing that the ambient cloudlets may be an important component for energy exchange between MM SNRs and their surroundings. However, it is clearly impossible to generalize from this one example to all objects in the class. The "dense medium" model could be suitable in other situations where the ambient interstellar medium is different from that around the Antlia SNR.

We note that, at an angular resolution of 2.5 , much of the sub-structure in the C IV map is almost certainly hidden. Thus, an FUV study of the Antlia SNR at much higher angular resolution (if and when that becomes possible) would allow the detailed structure of the radiatively cooling gas to be mapped and its relationship with the emission at other wavelengths to be characterized more precisely.

FIMS/SPEAR is a joint project of KASI \& KAIST (Korea) and U.C., Berkeley (USA), funded by the Korea MOST and NASA Grant NAG5-5355. The authors thank J. L. Jonas for the $2.3 \mathrm{GHz}$ radio map toward the Antlia SNR, and also appreciate valuable comments 
from the referee and B.-C. Koo.

\section{REFERENCES}

Borkowski, K. J., Balbus, S. A., \& Fristrom, C. C. 1990, ApJ, 355, 501

Chevalier, R. A. 1999, ApJ, 511, 798

Cowie, L. L., McKee, C. F., \& Ostriker, J. P. 1981, ApJ, 247, 908

Cox, D. P. \& Daltabuit, E. 1971, ApJ, 167, 113

Cox, D. P., Shelton, R. L., Maciejewski, W., Smith, R. K., Plewa, T., Pawl, A., \& Różyczka, M. 1999, ApJ, 524, 179

Danforth, C. W., Sankrit, R., Blair, W. P., Howk, J. C., \& Chu, Y.-H. 2003, ApJ, 586, 1179

Draine, B. T. 2003, ARA\&A, 41, 241

Edelstein et al. 2006a, ApJ, 644, L159

-. 2006b, ApJ, 644, L153

Edgar, R. J. \& Chevalier, R. A. 1986, ApJ, 310, L27

Finkbeiner, D. P. 2003, ApJS, 146, 407

Górski et al. 2005, ApJ, 622, 759

Hartigan, P., Raymond, J., \& Hartmann, L. 1987, ApJ, 316, 323

Jonas, J. L., Baart, E. E., \& Nicolson, G. D. 1998, MNRAS, 297, 977

Korpela et al. 2006, ApJ, 644, L163

Kregenow et al. 2006, ApJ, 644, L167

Kriss, G. 1994, in ASP Conf. Ser. 61: Astronomical Data Analysis Software and Systems III, 437

McCullough, P. R., Fields, B. D., \& Pavlidou, V. 2002, ApJ, 576, L41

Rho, J. \& Petre, R. 1998, ApJ, 503, L167

Seon et al. 2006, ApJ, 644, L175 
Shelton, R. L., Cox, D. P., Maciejewski, W., Smith, R. K., Plewa, T., Pawl, A., \& Różyczka, M. 1999, ApJ, 524, 192

Shinn, J.-H. 2007, PhD thesis, KAIST, Republic of Korea

Shinn et al. 2006, ApJ, 644, L189

Slavin, J. D. 1989, ApJ, 346, 718

Slavin, J. D. \& Cox, D. P. 1992, ApJ, 392, 131

Snowden et al. 1995, ApJ, 454, 643

Tilley, David A., Balsara, Dinshaw S., \& Howk, J. Christopher. 2006, MNRAS, 371, 1106

Weingartner, J. C. \& Draine, B. T. 2001, ApJ, 548, 296

White, R. L. \& Long, K. S. 1991, ApJ, 373, 543

This preprint was prepared with the AAS LATEX macros v5.2. 
Fig. 1.- The mixed-morphology of the Antlia SNR abutting on the Gum Nebula The RASS $3 / 4 \mathrm{keV}$ map (top-panel, Snowden et al. 1995) of the Antlia SNR is shown together with the contours of the Rhodes University/HartRAO $2.3 \mathrm{GHz}$ radio continuum map (Jonas et al. 1998). The radio features are seen as broken shells around the center-filled X-ray enhancement, especially at the bottom and right corners. The $\mathrm{H} \alpha$ map (bottom-panel, Finkbeiner 2003) shows a prominent arc feature along the faint radio shell. The Antlia SNR shows a simliar morphology with MM SNRs: the center-filled X-ray and shell-like radio morphology. The Antlia SNR abuts on the Gum Nebula at the lower-right corner. A circle of $24^{\circ}$ diameter, with its center at the core of the Antlia SNR, is shown on both maps. The coordinates are galactic. The units of colorbar are $10^{-6}$ counts $\mathrm{s}^{-1} \operatorname{arcmin}^{-2}$ and Rayleighs, respectively. 

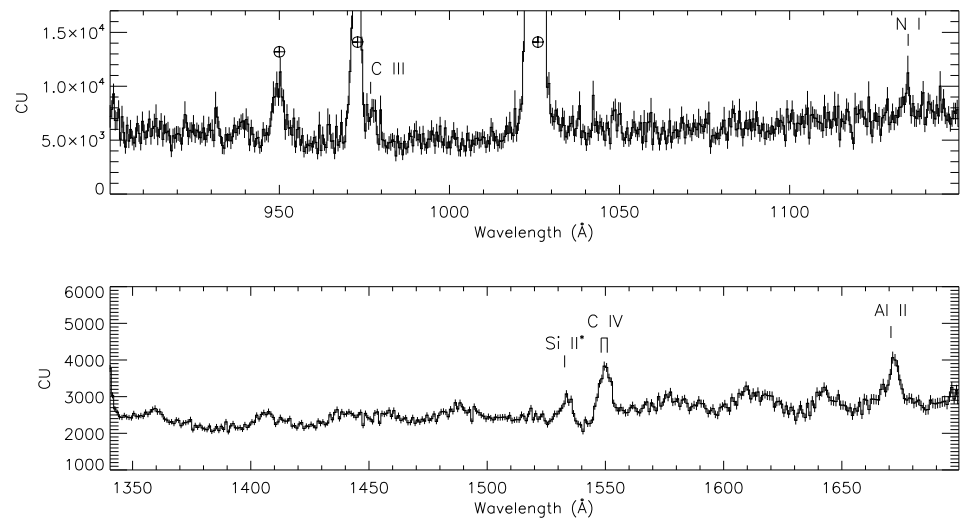

Fig. 2.- Emission lines identified from the total spectrum The total spectrum of each channel was extracted from the circle on Fig. 11. They are binned in $0.5 \AA$ and $1.0 \AA$ for the Short and Long channels, respectively. C III $\lambda 977$, N I $\lambda 1135$, Si II* $\lambda 1533$, C IV $\lambda \lambda 1548,1551$, and Al II $\lambda 1671$ are identified. ' $\oplus$ ' indicates the geocoronal lines. 
Fig. 3.- C IV $\lambda \lambda 1548,1551$ emission-line map The map was smoothed with a Gaussian kernel with 2.5 full-width-half-maximum. The unit of the colorbar is LU. The coordinates and the circles are the same as those in Fig. 1. The C IV map generally shows a clumpy distribution over the circle. The overlaid boxes inside the circle are the subregions where the spectra were extracted (cf. Fig. 4). The size of one box is $7.4 \times 2.0$. The "BG" box is the region where the intensity of the diffuse background C IV was estimated. It is $3620 \pm 724 \mathrm{LU}$. 

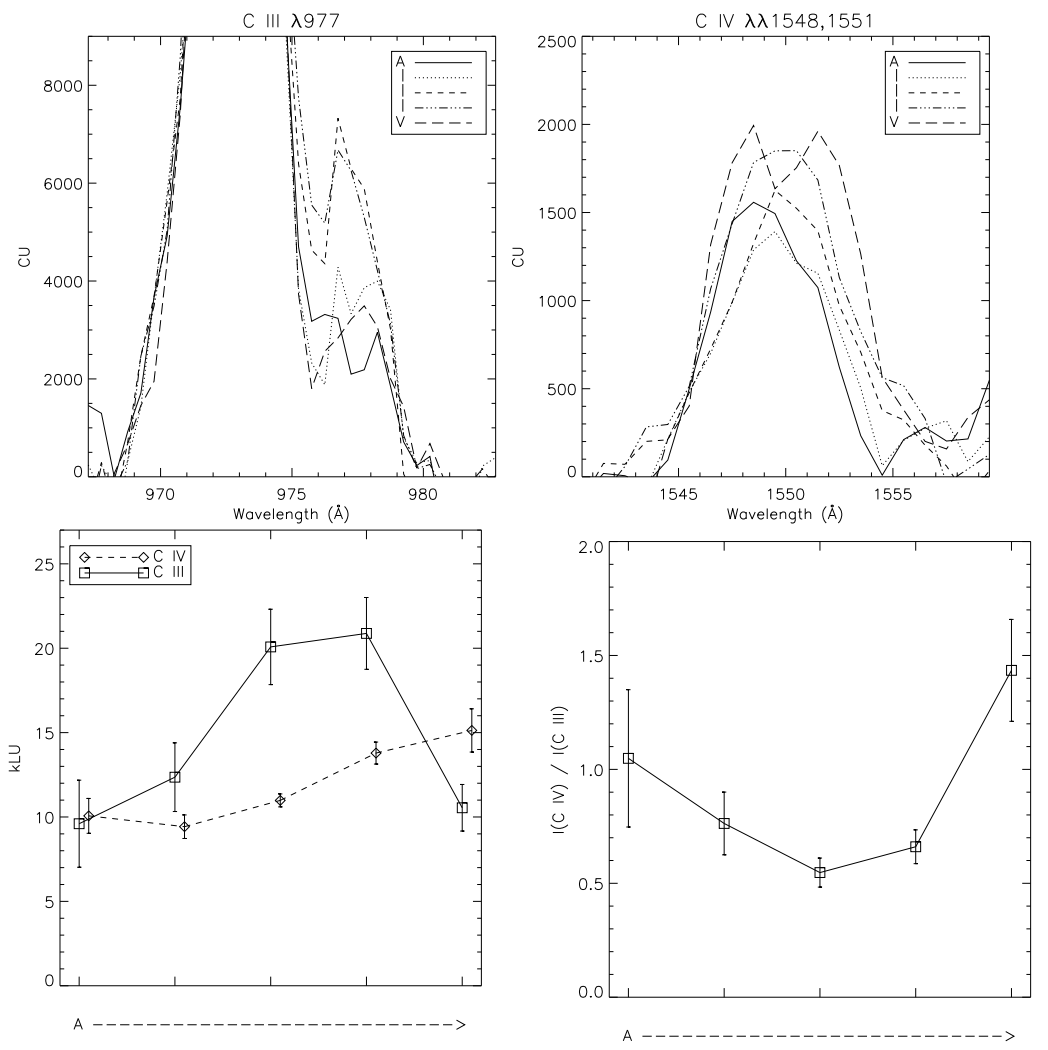

Fig. 4. - The radial variation of C III $\lambda 977$ and C IV $\lambda \lambda 1548,1551$ intensity The two upper panels are the spectra around C III $\lambda 977$ and C IV $\lambda \lambda 1548,1551$. They were extracted from two adjacent subregions - note that the number of spectra is five, while the number of subregions is six (see Fig. 3 and text). The lower-left panel plots the radial variations of their intensity. The C IV mark is slightly shifted to the right in the lower-left panel, to avoid the overlapping of the 1- $\sigma$ error bar. The lower-right panel plots the radial variation of the ratio $\mathrm{I}(\mathrm{C}$ IV $) / \mathrm{I}(\mathrm{C}$ III). The rise of $\mathrm{I}(\mathrm{C}$ IV $) / \mathrm{I}(\mathrm{C}$ III) near the edge suggests that the temperature is probably not decreasing outward. 
Table 1. Intensity of each identified emission line from the total spectrum

\begin{tabular}{cccc}
\hline \hline Emission Line & $\begin{array}{c}\text { Wavelength } \\
(\AA)\end{array}$ & $\begin{array}{c}\text { Observed } \\
\text { Intensity } \\
(\mathrm{LU})\end{array}$ & $\begin{array}{c}{ }^{\dagger} \text { Intrinsic } \\
\text { Intensity } \\
(\mathrm{LU})\end{array}$ \\
\hline C III & 977 & $8165 \pm 1864$ & $15669 \pm 3578$ \\
O VI & 1032,1038 & $<10483^{\ddagger}$ & $<19267^{\ddagger}$ \\
N I & 1134 & $6252 \pm 1480$ & $10609 \pm 2511$ \\
Si II & 1533 & $3582 \pm 403$ & $5231 \pm 588$ \\
C IV & 1548,1551 & $9891 \pm 526$ & $14399 \pm 766$ \\
Al II & 1671 & $6475 \pm 623$ & $9306 \pm 895$ \\
\hline
\end{tabular}

${ }^{\dagger}$ Extinction-corrected intensity with $N(\mathrm{H} \mathrm{I})=3.0 \times 10^{20}$ $\mathrm{cm}^{-2}$. See text.

$\ddagger 90 \%$ upper limit. 
This figure "f1a.jpg" is available in "jpg" format from: http://arxiv.org/ps/0710.1531v2 
This figure "f1b.jpg" is available in "jpg" format from: http://arxiv.org/ps/0710.1531v2 
This figure "f3.jpg" is available in "jpg" format from: http://arxiv.org/ps/0710.1531v2 